\title{
Design and implementation of a hot-wire probe for simultaneous velocity and vorticity vector measurements in boundary layers
}

\author{
S. Zimmerman ${ }^{1}$ - C. Morrill-Winter ${ }^{1}$ • J. Klewicki ${ }^{1,2}$
}

Received: date / Accepted: date

\begin{abstract}
A multi-sensor hot-wire probe for simultaneously measuring all three components of velocity and vorticity in boundary layers has been designed, fabricated and implemented in experiments up to large Reynolds numbers. The probe consists of eight hotwires, compactly arranged in two pairs of orthogonal $\times$-wire arrays. The $\times$-wire sub-arrays are symmetrically configured such that the full velocity and vorticity vectors are resolved about a single central location. During its design phase, the capacity of this sensor to accurately measure each component of velocity and vorticity was first evaluated via a synthetic experiment in a set of well-resolved DNS fields. The synthetic experiments clarified probe geometry effects, allowed assessment of various processing schemes, and predicted the effects of finite wire length and wire separation on turbulence statistics. The probe was subsequently fabricated and employed in large Reynolds number experiments in the Flow Physics Facility (FPF) wind tunnel at the University of New Hampshire. Comparisons of statistics from the actual probe with those from the simulated sensor exhibit very good agreement in trend, but with some differences in magnitude. These comparisons also reveal that the use of gradient information in processing the probe data can significantly improve the accuracy of the spanwise velocity measurement near the wall.
\end{abstract}

\footnotetext{
1 Department of Mechanical Engineering, University of Melbourne, Melbourne, VIC, Australia.

2 Mechanical Engineering Department, University of New Hampshire, Durham, $\mathrm{NH}$, USA.

S. Zimmerman

E-mail: zimmermans@unimelb.edu.au
}

To the authors' knowledge, the present are the largest Reynolds number laboratory-based measurements of all three vorticity components in boundary layers.

Keywords Vorticity · Hot-wire Anemometry · Wall Turbulence

\section{Introduction}

Fluctuating vorticity is a defining feature of all turbulent flows. Accordingly, the combined analysis of vorticity and velocity holds the potential to reveal fundamental properties of turbulence, e.g., see Tennekes and Lumley [1972]. The clear potential of such measurements is, however, contrasted with the capacity to attain them, especially at large Reynolds number. The experimental challenges associated with accurately measuring the velocity fluctuations and especially the vorticity vector fluctuations at large Reynolds number are well-known and considerable (e.g. see Wallace and Vukoslavčević [2010]). Owing to the influence of the no-slip condition on flow structure, these challenges are especially acute in turbulent boundary layers, e.g., Klewicki and Falco [1990].

As is the case for measurement of most turbulence quantities, there are two apparent ways to address the large Reynolds number measurement challenge. One is to develop new technologies to reduce the measurement volume and improve the frequency response of the sensor. The other is to generate a flow with physically larger (and lower frequency) dynamically relevant motions, such that these motions can be adequately resolved via existing sensor technology. The relatively recently developed Flow Physics Facility (FPF) at the University of New Hampshire [Vincenti et al., 2013] furthers the latter approach to simultaneous velocity/vorticity 
vector measurements at previously unattainable Reynolds number/spatial resolution combinations.

Even with the more favorable measurement environment afforded by the FPF, vorticity fluctuation measuring hot-wire probes are notoriously difficult to reliably deploy. These difficulties pertain to complex geometry effects (leading to both heat transfer and aerodynamic sources of uncertainty), variations in manufacturing process, calibration drift and the accuracy and appropriateness of the calibration devices, and the fragility of these probes. In connection with this, research in our group over the past decade has sought to mitigate a number of these challenges by developing significantly more repeatable manufacturing processes, e.g., grinding and plating procedures for the probe prongs, precision prong positioning jigs and procedures, and wire mounting techniques, a precision articulating jet calibration device, and improved data processing methods that take fuller advantage of the flow information provided by a multi-element probe [Ebner, 2014, Morrill-Winter, 2016, Morrill-Winter et al., 2015]. These have led to probes whose electrical properties are more predictable and stable, and whose physical robustness dramatically reduces mechanical failures of the probe wires and their connections to the prongs. Herein we report on the development of an 8 wire hot-wire probe that is tailored for measurements of the velocity and vorticity vectors in boundary layers, and present measurements from this sensor at the large Reynolds numbers provided by the FPF.

To the authors' knowledge, existing studies of turbulent boundary layers that present spatially or temporally resolved measurements of the full vorticity vector include the efforts of only a small number of research groups, as represented by the studies of Balint et al. [1987], Balint et al. [1991], Lemonis [1995], Lemonis [1997], Honkan and Andreopoulos [1997], Ong and Wallace [1998] and Ganapathisubramani et al. [2006]. These existing studies are cited extensively in this study, and so will be abbreviated as B87, B91, L97, HA97, OW98, and G06, respectively, from this point forward. Boundary layer measurements were also presented in Tsinober et al. [1992]. This study, however, contained results from only two positions in the wake region of the flow, as the primary focus was on grid turbulence. The data presented in all of these studies with the exception of G06 were acquired via multi-element hot-wire anemometry probes. The data presented in G06 were acquired via dual-plane stereo-PIV and thus include more spatial information than the hot-wire studies, but are not timeresolved and so lack the temporal information afforded by the present data. Other optical methods, such as single plane stereo PIV [Van Doorne and Westerweel,
2007], three-plane PIV [Zeff et al., 2003], and tomographic PIV [Schröder et al., 2008] have been used to measure the velocity gradient tensor in various flows, but none of these have been used to produce vorticity statistics in a turbulent boundary layer. As will become apparent by the data comparisons herein, previous studies that have presented vorticity statistics in turbulent boundary layers were performed at low Reynolds numbers, and are characterized by a relatively sparse measurement density per profile. The current effort employs a sensor design with a physical measurement volume and spatial resolution similar to previous studies, but is able to achieve Reynolds numbers up to a decade larger owing to the physical scale of the FPF. Furthermore, owing to the physical durability of the present sensor, much more detailed profile information is afforded by the larger number of points per profile in the present versus these previous studies.

B87, B91, and OW98 all used a version of the same 9 -wire sensor. Several flaws related to the version of this probe used in B87 were pointed out in B91 and corrected in the later two studies. B91 and OW98 acquired measurements in a boundary layers with a friction Reynolds numbers of $\delta^{+}=\delta u_{\tau} / \nu \approx 1100$ and $\delta^{+} \approx 545$, respectively. Here $\delta$ is the boundary layer thickness, $\nu$ is the kinematic viscosity, $u_{\tau}$ is the friction velocity $\left(=\sqrt{\tau_{w} / \rho}\right), \tau_{w}$ is the mean wall shear stress, and $\rho$ is the mass density. The sensor sub-array separation (the distance over which gradients were estimated) was approximately 11 viscous lengths in both the spanwise and wall-normal directions in B91, compared to 7 in OW98. Streamwise gradients for this study, as well as for all other previous hot-wire studies, were estimated via Taylor's hypothesis.

The measurements presented in HA97 were acquired in a turbulent boundary layer with a friction Reynolds number of $\delta^{+} \approx 1200$. The sub-array separation of the probe used in this study was approximately 21 viscous lengths in the wall-normal direction, and 18 viscous units in the spanwise direction.

L97 improved upon the technique of L95 with slight modifications to the 20 -wire probe originally used in the earlier study. The second of these two studies presented data acquired in a turbulent boundary layer with friction Reynolds number of $\delta^{+} \approx 2300$. This sensor had a total sub-array separation of approximately 21 viscous lengths in both the spanwise and wall-normal directions, and featured 5 sub-arrays capable of measuring the full velocity vector, whereas the probes used by B91 and HA97 featured 3 sub-arrays. The use of two additional sub-arrays allowed for a second-order estimation of the velocity field, as well as the symmetric estimation of all velocity gradients about a common location. 
While the probes and data reduction methods employed by B91 and HA97 also produce both the velocity and vorticity about a common location, the gradient estimates about that location are based on measurement points that are not symmetric. The advantages of a symmetric design are noted in Vukoslavčević and Wallace [2013], and are related to the reduction in error associated with central differencing as opposed to forward or backward differencing.

This study presents data acquired in turbulent boundary layers with friction Reynolds numbers ranging from $\delta^{+} \approx 2600$ to 13200 . Owing to the presence of mean shear in the turbulent boundary layer, the sub-arrays have been arranged to minimize the wall-normal spacing, but at the expense of a larger spanwise spacing. Note that aspects of the present probe design concept, including the prioritization of $x_{2}$ gradient spacing, stem from conversations with J. Wallace. The spatial resolution associated with the individual velocity components ranges from 13 to 25 viscous lengths over the $\delta^{+}$ range reported herein. The distances over which gradients are computed in this study range from 13 to 25 viscous lengths in the wall-normal direction, and 33 to 63 viscous lengths in the spanwise direction. While these larger than desired separations lead to significant attenuation of the vorticity signals in the near-wall region at large $\delta^{+}$, it will be shown that there is much less attenuation of the motions within the inertial logarithmic layer and wake region.

In the following sections, the effects of finite spatial resolution on the performance of the current probe are quantified using a synthetic probe experiment that is based on the interrogation of the turbulent boundary layer DNS data set of Sillero et al. [2013]. This synthetic experiment has similarities to that presented in Vukoslavčević et al. [2009]. Besides incorporating additional features (such as finite wire length effects), the present approach designed, fabricated and used an actual sensor based upon features learned from the synthetic probe experiments. In the context of the resolution limitations predicted by this synthetic experiment, results from four turbulent boundary layer profile scans are presented and discussed. Overall, while spatial attenuation of the velocity gradients is apparent in the present measurements, it is also apparent that key features of the vorticity are captured.

\section{Methodology}

This section includes a description of the facility, the probe and its design, and the various processing schemes used to obtain the statistics presented in section 4 . In what follows, the streamwise, wall-normal, and spanwise directions are denoted with the subscripts 1, 2, and 3 , respectively. $U$ and $u$, for example, represent the total and fluctuating streamwise velocity, while overbar notation indicates a time-averaged quantity. Vorticity is denoted by variants of $\omega$, with the subscript indicating the vorticity component direction.

\subsection{Flow Physics Facility}

All of the measurements presented herein were collected at the Flow Physics Facility at the University of New Hampshire. This facility is designed for large Reynolds number measurements at high spatial resolution, as it produces friction Reynolds numbers in excess of $10^{4}$ with viscous length scales as large as $60 \mu \mathrm{m}$. These features stem from its combination of low speed stability and long streamwise development length $(>60 \mathrm{~m})$. This is particularly important for gradient measurements, as velocity gradient fluctuations concentrate over a higher wavenumber range than that of the velocity fluctuations. Thus, these measurements require even finer spatial resolution than is typically acceptable for the measurement of velocity fluctuations. A characterization of the FPF can be found in Vincenti et al. [2013], and previous $u_{1}, u_{2}$ and $\omega_{3}$ measurements from the FPF are reported in Morrill-Winter et al. [2015].

\subsection{Enstrophy Probe}

As discussed in section 1, only a few studies have presented time-resolved data of all three components of vorticity in boundary layers. In these cases, however, the probes employed actually measured the entire velocity gradient tensor rather than specifically the vorticity vector. For the full velocity gradient tensor, measurement of both the normal and shear velocity gradients necessitates a probe design with at least 9 wires, as with B87, B91, OW98, and HA97. Some probes, such as those of L95 and L97 have used as many as 20 wires. Velocity gradient tensor probes, for practical reasons, also require sub-arrays capable of resolving all three velocity components each. If, however, the focus is only on the velocity and vorticity vectors, a probe may be constructed with as few as 8 wires. This 8 -wire style of probe, a variant of which was first developed by Antonia et al. [1998], need not be composed of sub-arrays that each measure all three components of velocity. Reducing the number of velocity components measured by each sub-array from three to two facilitates a more rugged probe design, while significantly reducing the calibration time and processing complexity. The merits 
of targeting just two velocity components per sub-array are evidenced by the quality of the present velocity variance data.

The present probe geometry is depicted in figure 1. As with the probes employed in Antonia et al. [1998] and Zhou et al. [2003], this probe consists of 8 sensing elements, of which 4 are parallel to the $x_{1}-x_{2}$ plane and 4 are parallel to the $x_{1}-x_{3}$ plane. Unlike these previous sensors, the present design positions the horizontally (wall-parallel) oriented wires closer together, at the expense of positioning the outboard pairs of $\times$-arrays farther apart in the spanwise direction. This attribute was chosen, and reinforced from our synthetic probe experiments, to provide better spatial resolution for the wallnormal $\left(x_{2}\right)$ derivatives. For the probe depicted in figure 1 , the 4 elements in the $x_{1}-x_{2}$ plane yield the $u_{1}$ and $u_{2}$ components of velocity along with their $x_{3}$ gradients, while the 4 in the $x_{1}-x_{3}$ plane yield the $u_{1}$ and $u_{3}$ components of velocity along with their $x_{2}$ gradients. Taylor's frozen turbulence hypothesis is used with the local mean velocity to estimate streamwise gradients.

The present sensing elements are $5 \mu \mathrm{m}$ diameter platinum-plated tungsten wire, with a length projected onto the $x_{2}-x_{3}$ plane of $l_{w_{p}}=0.8 \mathrm{~mm}$, and an overall length of $l_{w}=l_{w_{p}} \sqrt{2} \mathrm{~mm}$. This constitutes a wire length-to-diameter ratio in excess of 225 , which satisfies the criteria of Ligrani and Bradshaw [1987] to mitigate the effects of conductive heat loss. In lieu of wire 'stubs' to further mitigate end conduction, the $5 \mu \mathrm{m}$ sensing elements are soldered directly to copper-plated steel support prongs, each with a tip diameter of $50 \mu \mathrm{m}$. This feature allows for a significant reduction in measurement volume compared to the stubbed-wire probes used in Antonia et al. [1998] and Zhou et al. [2003], and subsequently increases the maximum achievable spatial resolution. The larger wire diameter and higher tensile strength of tungsten also significantly increases the durability of the probe relative to the $2.5 \mu \mathrm{m}$ platinumrhodium used in those studies.

Antonia et al. [1998] described their arrangement as an array of 4 individual $\times$-wire probes. While this description is apt for their probe and processing scheme, it does not apply to the current method of processing. This distinction will be discussed in detail in section 2.4. For illustrative purposes, however, the $4 \times$-array picture is useful for understanding the operating principles of the probe, and, as reflected by the indicated measurement locations, is used in figure 1. In this conceptual picture, independent velocity measurements are collected at the positions indicated by the dots in figure 1 , and $x_{2}$ and $x_{3}$ gradients are estimated by finite differences. Note that these estimates are central differences about the probe centroid, which afford a higher degree of accuracy than a forward/backward or otherwise asymmetric difference scheme. The full vorticity vector is resolved about the centroid of the probe using the following equations:

$$
\begin{aligned}
& \omega_{1}=\frac{\partial u_{3}}{\partial x_{2}}-\frac{\partial u_{2}}{\partial x_{3}} \approx \frac{u_{3_{a}}-u_{3_{b}}}{\Delta x_{2}}-\frac{u_{2_{c}}-u_{2_{d}}}{\Delta x_{3}} \\
& \omega_{2}=\frac{\partial u_{1}}{\partial x_{3}}-\frac{\partial u_{3}}{\partial x_{1}} \approx \frac{u_{1_{a}}-u_{1_{b}}}{\Delta x_{3}}-\frac{u_{3_{a}}+u_{3_{b}}}{2 U_{c} \Delta t} \\
& \omega_{3}=\frac{\partial u_{2}}{\partial x_{1}}-\frac{\partial u_{1}}{\partial x_{2}} \approx \frac{u_{2_{c}}+u_{2_{d}}}{2 U_{c} \Delta t}-\frac{u_{1_{a}}-u_{1_{b}}}{\Delta x_{2}} .
\end{aligned}
$$

The full velocity vector is also resolved about the centroid of the probe by averaging (at each instant) the appropriate measurements.

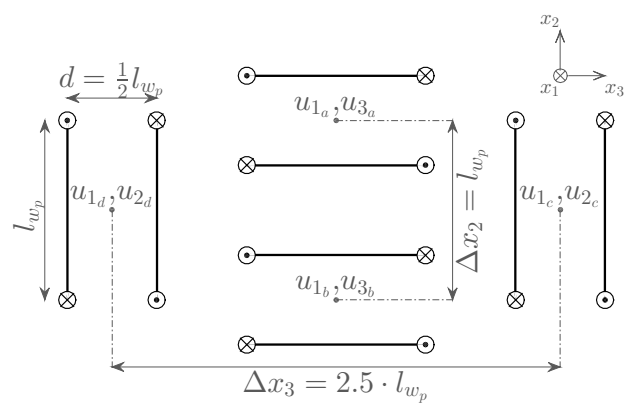

Fig. 1 Front view of the present probe geometry. Dimensions are based on $l_{w_{p}}$, the wire length projected onto the $x_{2}-x_{3}$ plane. Prong locations marked by $\odot$ are at $x_{1}=-\frac{1}{2} l_{w_{p}}$ and those marked by $\otimes$ are at $x_{1}=+\frac{1}{2} l_{w_{p}}$ such that each wire is inclined at $45^{\circ}$ to the mean flow. Velocity measurement locations are based on a $\times$-wire processing scheme.

\subsection{Acquisition}

Each wire was operated by one of eight identical custom built Melbourne University Constant Temperature Anemometer (MUCTA) channels. The output of each channel was routed through an Alligator Technologies USBPGF-S1 programmable analog low pass filter. The inner-normalized cutoff frequency for each measurement exceeded 1 . The channels were acquired along with ambient temperature and free-stream dynamic pressure using a Data Translation DT9836 12-channel 15-bit signed simultaneous A/D board. Ambient pressure was recorded manually at regular time intervals throughout the experiment. 


\subsection{Processing}

A number of variations of the chosen processing scheme were considered. Here we describe the one we chose, along with a comparison to a standard $\times$-wire processing scheme. Traditional $\times$-array processing schemes yield estimates of two velocity components from the output of the two sensing elements. Since this estimate is presumed to apply at the centroid of the array (as shown in figure 1), any non-uniformity in the incident flow between the constituent sensing elements can result in significant errors, e.g. see Park and Wallace [1993]. This is of particular concern when measuring wall-bounded flows, because of the large nearwall mean gradient, $\partial U_{1} / \partial x_{2}$. Multi-wire probes have an advantage over a single $\times$-array probe in this respect because they are designed to measure these same gradients, and thus the measured gradient estimates can be used to mitigate the effect of the error. Below we present results, in 2.4.2 and 2.4.3, from processing the raw probe output in two ways. These demonstrate a check for consistency, and provide estimates regarding the influence of gradient aliasing.

\subsubsection{Calibration}

Both processing schemes require information on pitch, yaw, and velocity magnitude sensitivity for each wire. This information was extracted from a calibration following the same procedure used in the pitch-only calibrations of Morrill-Winter et al. [2015]. A bespoke computer controlled articulating jet was used to measure the response of each wire to pitch and yaw angles of $-30^{\circ}$ to $30^{\circ}$ (relative to the flow coordinate system) across a range of velocities corresponding to those expected in the given experiment. Jet calibrations were performed before and after each profile measurement, with each calibration consisting of at least 8 velocities, and each velocity consisting of 13 pitch and 13 yaw measurements. The response of each wire was found to be well described by the formulation in equation 5, first suggested by Jorgensen [1971].

$$
\begin{aligned}
e & =f\left(u_{e}\right) \\
u_{e}^{2} & =u_{n}^{2}+k^{2} u_{t}^{2}+h^{2} u_{b}^{2}
\end{aligned}
$$

Equation 4 introduces the 'effective' cooling velocity, which by its construction completely determines the sensor output. For the present analysis, $f$ is constructed by first fitting the calibration data to a third-order polynomial of $e$ to determine $u_{e}$, and then fitting a spline through this polynomial so that it may be inverted. This process is done for calibration data acquired before and after each experiment. The calibration curve used to process each profile location is generated by interpolating between these two calibrations using the measured ambient temperature.

Equation 5, more commonly known as Jorgensen's expression, states that any incident velocity vector can be resolved into an 'effective' cooling speed by splitting it into components along the directions normal, tangential, and bi-normal to the sensing wire axis, and accounting for the difference in cooling efficiency in each direction with the coefficients $k$ and $h$. While other models of wire response have been proposed (e.g. see Dobbeling et al. [1990]), the fundamental tasks of the model (when the input data are free from noise) are to fit the calibration data, and reduce the error associated with interpolation between calibration points. When the density of calibration points is sufficiently high, all that is required of a functional form is that it produces a smooth fit of the data with minimal squared-error. The process suggested by Dobbeling et al. [1990], for example, is better equipped to account for asymmetry in sensor response compared to Jorgensen's expression, and so for certain probe designs provides a superior fit. For the present case, however, Jorgensen's expression produces a satisfactory fit of the calibration data, which, as shown in figure 2, are densely packed and vary smoothly. Thus, there is little potential for error associated with response asymmetry and/or interpolation. The remaining terms in equation 5 are written in the flow coordinate system in equation 6 , where $u_{x}$ is either $u_{2}$ or $u_{3}$ depending on the wire orientation, and $\alpha$ is the 'effective' angle [Bradshaw, 1971] between the wire and the positive streamwise axis in the sensor plane.

$u_{e}^{2}=\left(u_{1} \sin \alpha-u_{x} \cos \alpha\right)^{2}+k^{2}\left(u_{1} \cos \alpha+u_{x} \sin \alpha\right)$

By subjecting the sensors to a range of $\left(u_{1}, u_{x}\right)$ velocity combinations, the coefficients $k$ and $\alpha$ can be determined for each wire from a least squares fit, along with the coefficients for the chosen function $f\left(u_{e}\right)$ from equation 4. In keeping with the findings of Lawson and Britter [1983] and Snyder and Castro [1998] (that $k$ and $\alpha$ are velocity-dependent), and following the recommendation of Bakken and Krogstad [2004], the coefficients $k$ and $\alpha$ are determined separately at each calibration speed, and then treated as a function of speed in both of the processing schemes described herein. Upon acquiring numerous calibration data sets with the present sensor, however, it was found that the dependencies of $k$ and $\alpha$ on flow speed are very weak for velocities above about $3\left[\frac{\mathrm{m}}{\mathrm{s}}\right]$. Consideration of the sensitivity of each wire to bi-normal cooling had very little effect on any of the statistics presented herein. Thus, for the present analysis, the effect of the bi-normal velocity term $h^{2} u_{b}^{2}$ is neglected. This insensitivity to bi-normal cooling is 
not surprising based on its role in the governing equations. Since both sensing elements in a $\times$-array are approximately equally sensitive to bi-normal cooling, binormal velocity should increase the signal from both wires by the same amount. A symmetric increase in signal from both sensors should influence only the $u_{1}$ component rather than the $u_{2}$ or $u_{3}$ components, as the former is essentially dependent on the sum of the two wire outputs, and the latter on the difference between the two wire outputs. It therefore stands to reason that bi-normal cooling has very little effect on the reduced data, as the magnitudes of the cross-stream components $u_{2}$ and $u_{3}$ are typically much smaller than $u_{1}$ in the present flow and measurement domain, and are thus unlikely to cool the wire enough to dramatically affect the $u_{1}$ output.

\subsubsection{Four independent $\times$-wire arrays}

Under this processing scheme, two-dimensional lookup tables adhering to the functional form of equations 4 and 5 are constructed using the coefficients extracted from the articulating jet calibration. Unique lookup tables resembling those in figure 2 are generated for each pair of orthogonal coplanar wires. Velocity time series are interpreted for each pair based on the recorded voltage time series, and estimates of vorticity are made according to equations 1-3.

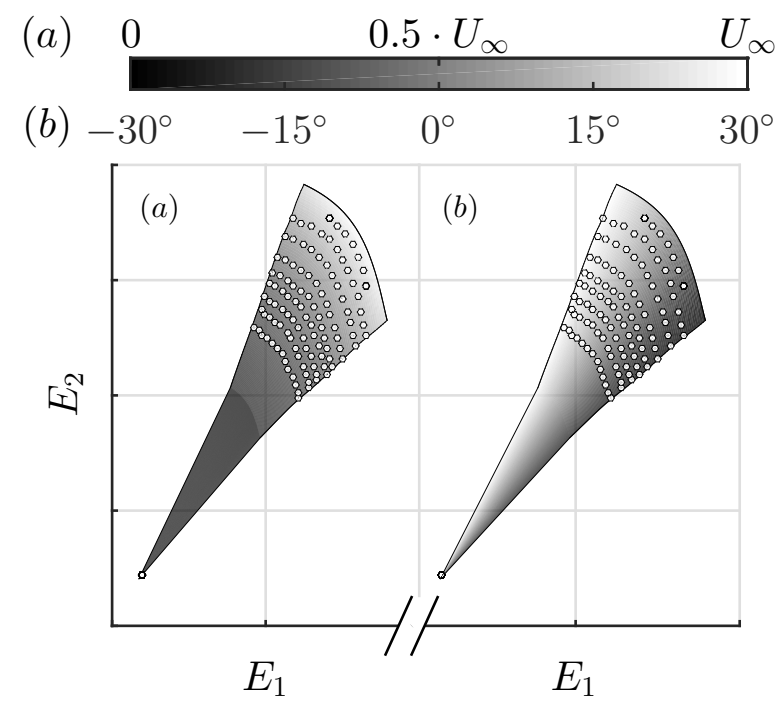

Fig. 2 Surface representation of typical lookup table set. Sample probe output given by $\circ$. (a) Table for streamwise velocity $u_{1}$. (b) Table for pitch/yaw angle $\theta_{x}=\arctan \left(u_{x} / u_{1}\right)$

\subsubsection{Two 4-wire 'gradient arrays'}

This processing scheme leverages the additional information provided by a multi-wire probe. As introduced in section 2.4, a necessary assumption of a single $\times$ wire probe is that the flow is uniform across the measurement volume. While this assumption increases in validity as the spatial scale of the probe diminishes, (or equivalently, as the velocity gradients across the wires diminish), it is undoubtedly suspect when the object is to measure the gradient over a distance that is similar to that over which the flow variations are supposed to be negligible. The information provided by the additional sensors can be exploited to replace the uniform velocity assumption with one that approximates flow variations as uniform gradients. Velocity and gradient information are recovered simultaneously by solving two sets of fourequation systems of the form given by equations 7-8. Note that in these equations the shorthand $s_{j}=\sin \alpha_{j}$ and $c_{j}=\cos \alpha_{j}$ has been used.

System 1: (wires $j=1-4$ in $x_{1}-x_{3}$ plane)

$$
\begin{gathered}
u_{e_{j}}^{2}=\left[\left(u_{1}+h_{j} \frac{\partial u_{1}}{\partial x_{2}}\right) s_{j}-\left(u_{3}+h_{j} \frac{\partial u_{3}}{\partial x_{2}}\right) c_{j}\right]^{2} \ldots \\
k_{j}^{2}\left[\left(u_{1}+h_{j} \frac{\partial u_{1}}{\partial x_{2}}\right) c_{j}+\left(u_{3}+h_{j} \frac{\partial u_{3}}{\partial x_{2}}\right) s_{j}\right]^{2}
\end{gathered}
$$

System 2: (wires $j=5-8$ in $x_{1}-x_{2}$ plane)

$$
\begin{gathered}
u_{e_{j}}^{2}=\left[\left(u_{1}+h_{j} \frac{\partial u_{1}}{\partial x_{3}}\right) s_{j}-\left(u_{2}+h_{j} \frac{\partial u_{2}}{\partial x_{3}}\right) c_{j}\right]^{2} \ldots \\
k_{j}^{2}\left[\left(u_{1}+h_{j} \frac{\partial u_{1}}{\partial x_{3}}\right) c_{j}+\left(u_{2}+h_{j} \frac{\partial u_{2}}{\partial x_{3}}\right) s_{j}\right]^{2}
\end{gathered}
$$

The systems represented by equations 7 and 8 are solved for each measurement instant using the results obtained by the method described in section 2.4.2 as initial guesses.

\section{Synthetic Experimental Validation}

A synthetic probe experiment was developed through the use of the boundary layer DNS data set of Sillero et al. [2013]. The results from these experiments aided in design decisions pertaining to the physical probe's prong positions, as well as in better understanding the ramifications of specific wire arrangements relative to spatial resolution. Once the probe was constructed and deployed, the synthetic experimental results allowed an evaluation of the actual performance relative to the predicted performance. To the extent that spatial attenuation scales with viscous units (independent of Reynolds number), the synthetic experiment also allows prediction of the effects of attenuation on measurements at 
higher Reynolds numbers generated by increasing $u_{\tau} / \nu$. These results are described below. The present approach to sensor evaluation, as well as the means by which the predicted output is computed, follow a similar methodology as that described in Vukoslavčević et al. [2009]. To the authors' knowledge, the present probe design and evaluation is the first to be aided by DNS-based synthetic experiments from inception.

In the synthetic experiments, average velocity is computed using the DNS field interrogated at several points along each sensing element. These average velocities are passed to equation 5 along with typical values for $k$ and $h$ of 0.2 and 1.05 [Bruun, 1995] to obtain effective cooling velocities (or, equivalently, wire voltages). Velocity is then recovered in the same way as described in section 2.4. This approach seeks to emulate the process by which velocity is interpreted by the sensor in a physical experiment, and is distinct from a simple box filter across the measurement volume. The current effort expands upon the methods of Vukoslavčević et al. [2009] by considering the effects of finite sensor length (in addition to sensor separation), and non-ideal but empirically consistent cooling coefficients $k$ and $h$ of 0.2 and 1.05 (as opposed to 0 and 1), respectively. The output predicted by this method includes the effects of: finite sensor length and measurement volume, sensorseparation-related gradient error, the error caused by neglecting the bi-normal component of velocity, and the distribution of error amongst the various terms in equations 7 and 8 when the tangential cooling coefficient $k$ is non-zero. Not accounted for by this model are the potential effects of: aerodynamic blockage of prongs and/or probe body, thermal crosstalk between sensor elements, deviations in convection velocity from the local mean velocity, conductive heat transfer to supporting prongs, conductive heat transfer to the bounding wall, uncertainty in calibration, frequency response, sensor drift, and electrical noise.

\subsection{Performance of processing methods}

The synthetic experiment code allows, for example, a direct performance evaluation of the two processing schem described in section 2.4. The correlation between the output of each scheme and the known flow input (for the measured velocities and gradients) is used to gauge effectiveness, and for the two schemes described herein is shown in figure 3 .

The processing scheme that treats the sensor as two 4 -wire 'gradient arrays' improves the velocity measurements for all three components across the entire boundary layer when compared to the scheme that treats the sensor as 4 independent $\mathrm{x}$-wire arrays. Note that the $u_{2}$ velocity component $\times$-array curves represent the accuracy of the average of the outputs of sub-arrays $c$ and $d$ from figure 1 , since this is necessary to obtain the measurement at the centroid of the probe. The accuracy of the $u_{2}$ component measured from an individual subarray is associated with the centroid of that particular array, not with the centroid of the probe. When the object is to measure the $u_{2}$ component at the centroid of the probe, the average of sub-arrays $c$ and $d$ is superior to either sub-array individually, despite the latter being significantly less spatially attenuated.

The $u_{3}$ component $x$-array curves are obtained from processing the two central wires as an independent $\times$ array. The largest improvement by incorporating gradient information is realized in the $u_{3}$ signal. This is not surprising, as this component is susceptible to error induced by the (relatively strong) $\partial u_{1} / \partial x_{2}$ gradient inherent to boundary layer flow, [Vukoslavčević and Wallace, 1981]. The choice of the gradient-array scheme seems to have very little effect on the accuracy of the gradients themselves for this probe orientation, apart from a near-wall improvement in $\partial u_{1} / \partial x_{2}$. Had the measurement elements been arranged such that the two central wires were in the same orientation rather than orthogonal, this effect may have been reversed. That is, since $\times$-arrays of the same orientation will produce the same error when exposed to a constant gradient, a quantity based on the difference between the two arrays will be unaffected. Thus, reversing the orientation of one sub-array would likely shift the aliasing error from the average velocity signal to the gradient estimation.

\section{Physical Experimental Results}

This section includes statistics acquired from physical measurements collected in the FPF. These are plotted alongside existing data from the studies described in section 1 as well as results predicted by the synthetic esexperimental validation described in section 3 . Both the physical and synthetic experimental data reflect the results of processing with the gradient-array approach. The current and existing physical data as well as comparisons with synthetic experiments are summarized in table 1. Note that the data from B87 and L95 have not been included as these studies were later succeeded by B91 and L97, respectively. Data from OW98 also have not been included, as they were acquired at a very low Reynolds number with the same probe as was used in B91. 

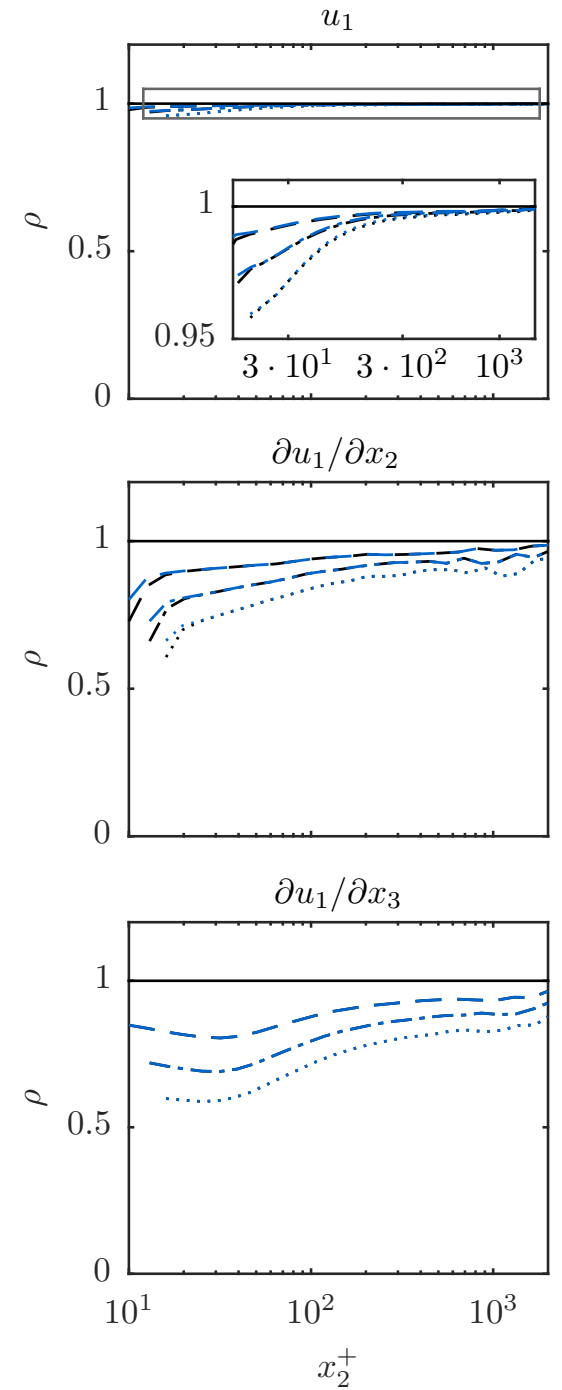
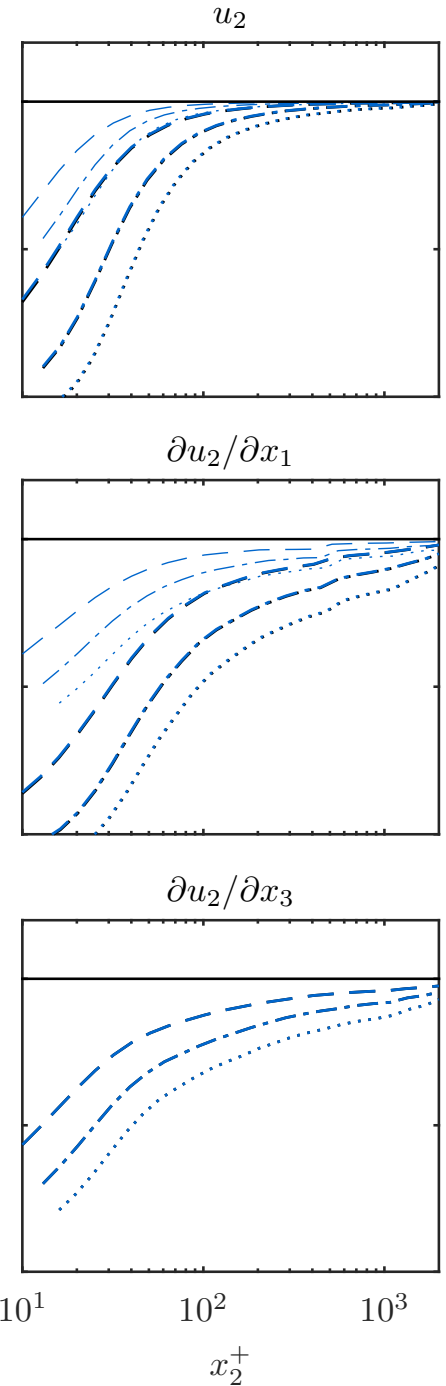
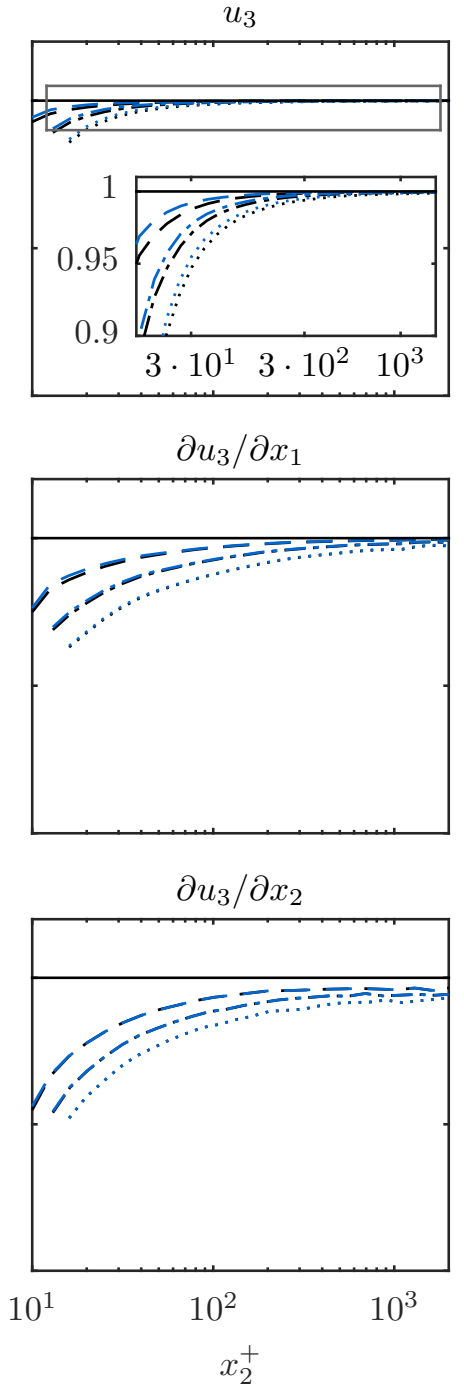

Fig. 3 Correlation between predicted probe output and DNS flow input for various measured quantities. Black lines represent results obtained by processing the sensor as four independent $\times$-wire arrays. Blue lines represent results obtained by processing the sensor as two 4-wire gradient-arrays. Dashed lines, dash-dotted lines, and dotted lines represent inner-normalized wire lengths of $l^{+}=14,20$, and 25, respectively. Thin lines in the $u_{2}$ and $\partial u_{2} / \partial x_{1}$ plots represent results for a single sub-array (c or $d$ from figure 1), while thicker lines represent results for the average $u_{2}$ signal about the probe centroid.

\subsection{Velocity Statistics}

Mean streamwise velocity and variances of each velocity component are plotted in figures 4 to 7 . The experimental mean velocity data exhibit the expected logarithmic behavior between the buffer and wake regions. The friction velocity $u_{\tau}$ was determined by fitting each profile according to the composite method suggested by Chauhan et al. [2009], where the reciprocal of the slope, $\kappa$, and the intercept $A$ are taken as 0.39 and 4.3, respectively [Marusic et al., 2013]. This fit causes the four profiles to merge from the logarithmic region out- ward. The tendency of this probe to slightly underestimate the mean velocity close to the wall is predicted by the DNS model and observed in the experimental data, with general agreement in trend with spatial resolution.

The streamwise velocity variance, plotted in figure 5 , explicitly shows good agreement between the experiment and the simulation for $\delta^{+}$close to that of the DNS. The observed deviations at larger $\delta^{+}$are expected, as the peak variance grows with $\delta^{+}$particularly owing to increased contributions from large scales, (e.g. see Metzger and Klewicki [2001], Hutchins and Marusic [2007]) 


\begin{tabular}{|c|c|c|c|c|c|}
\hline & Method & $u_{\tau}\left[\frac{\mathrm{m}}{\mathrm{s}}\right.$ & $\delta_{99}[\mathrm{~m}]$ & $\delta^{+}[-]$ & $l^{+}[-]$ \\
\hline$\nabla$ & 8-w HWA & 0.16 & 0.21 & 2600 & 14 \\
\hline ם & 8-w HWA & 0.15 & 0.63 & 7300 & 13 \\
\hline 。 & 8-w HWA & 0.22 & 0.62 & 11000 & 19 \\
\hline$\Delta$ & 8-w HWA & 0.29 & 0.60 & 13200 & 25 \\
\hline & DNS & - & - & 2000 & - \\
\hline-- & Synth. Exp. & - & - & 2000 & 14 \\
\hline$-\cdot-$ & Synth. Exp. & - & - & 2000 & 20 \\
\hline ....... & Synth. Exp. & - & - & 2000 & 25 \\
\hline & DNS & - & - & 1300 & - \\
\hline मे & 9-w HWA & 0.14 & 0.13 & 1100 & 6 \\
\hline 4 & 9-w HWA & 0.13 & 0.14 & 1200 & 8 \\
\hline$\diamond$ & 20-w HWA & 0.23 & 0.16 & 2300 & 8 \\
\hline$\otimes$ & 2-plane PIV & 0.25 & 0.07 & 1200 & $\dagger$ \\
\hline
\end{tabular}

Table 1 Summary of present and existing physical experiments as well as present synthetic experiments. Marker fill colors vary by processing case, but shapes consistently represent the same profiles. Turbulent boundary layer DNS from Sillero et al. [2013], and all synthetic experimental results calculated using available fields from the same data set. refers to B91, refers to HA97, $\diamond$ refers to L97, and $\otimes$ refers to G06. $\dagger$ Inner-normalized resolution based on window size in $x_{1}$ and $x_{3}$, and laser-sheet separation in $x_{2}: x_{i}^{+}=(24,20.5$, 24).

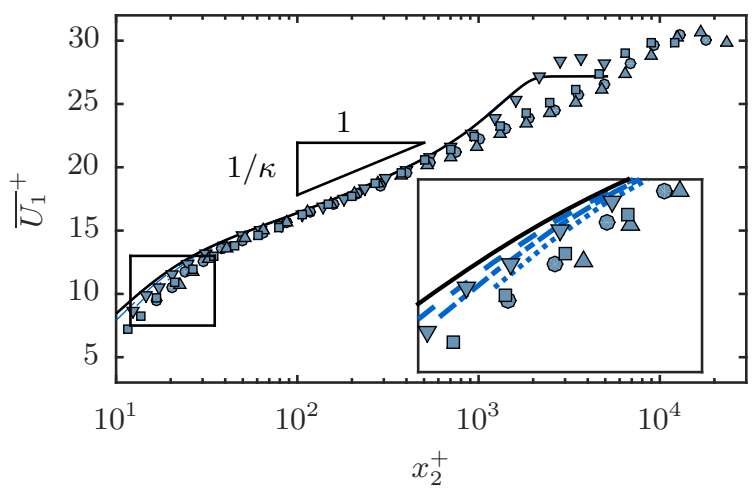

Fig. 4 Inner-normalized mean velocity profiles. Symbols are given in table 1. Inset shows enhanced view of near-wall region to highlight spatial resolution trends. The depicted slope is calculated using $\kappa=0.39$ [Marusic et al., 2013].

whose measured contribution to the variance is not significantly reduced by the decreasing spatial resolution of the small scales. Indeed, the measured inner peak variance of the $\delta^{+} \approx 7300$ case is considerably larger than that of the $\delta^{+} \approx 2600$ case at approximately matched $l^{+}$. Regarding these results, and the other velocity variances, it is worth noting that, while the effects of spatial resolution are detectable, they still constitute some of the largest $\delta^{+}$(yet well-resolved) boundary layer measurements in existence.

Of the comparison studies described in section 1 and summarized in table 1, only B91 and HA97 presented statistical moments of the velocity vector components measured with the same probe that was used to acquire the vorticity vector. Direct comparison of the stream- wise velocity variance between these two studies and the present data is difficult owing to the difference in Reynolds number. Thus, a DNS result corresponding to $\delta^{+} \approx 1300$ (taken from the same DNS data set as the $\delta^{+} \approx 2000$ curve) has been added to figure 5 as a reference for the comparison data. The near-wall peak in the B91 data is approximately in the same location and of the same magnitude as the peak in the lowest Reynolds number profile of the present data. The streamwise velocity fluctuation data presented in HA97 do not contain a discernible near-wall peak.

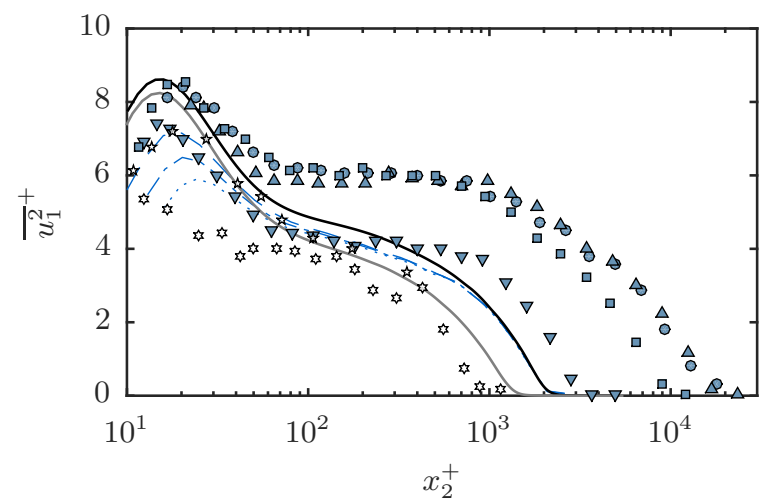

Fig. 5 Inner-normalized existing and present streamwise velocity variance data acquired by probes which simultaneously measure velocity and vorticity vectors. Symbols and lines as in table 1.

In contrast to the $\delta^{+}$dependence exhibited by the streamwise velocity variance, the measured inner-normalized wall-normal variance closely matches the simulation predictions interior to the wake region for all Reynolds numbers. Attached eddy modeling concepts [Townsend, 1976] predict this $\delta^{+}$invariance, and consistently, wellresolved hot-wire measurements of the wall-normal velocity [Morrill-Winter et al., 2015] show little to no dependence on $\delta^{+}$in the logarithmic region. This may, in part, explain the better agreement between the simulated and actual results than observed for the streamwise velocity variance.

The two sets of symbols in figure 6 represent the signal obtained from a single sub-array (dark fill), and the average of the two sub-arrays (light fill). The variance of the average signal between the two arrays has clearly suffered significant attenuation close to the wall relative to the individual sub-array signal. This average signal is, however, most appropriate for computing the $\partial u_{2} / \partial x_{1}$ component of $\omega_{3}$ (and velocity-vorticity correlations) since it is resolved about the center of the measurement volume. 
The present lowest Reynolds number wall-normal velocity fluctuation data exhibit good agreement with the data presented in B91, but not with the data presented in HA97.

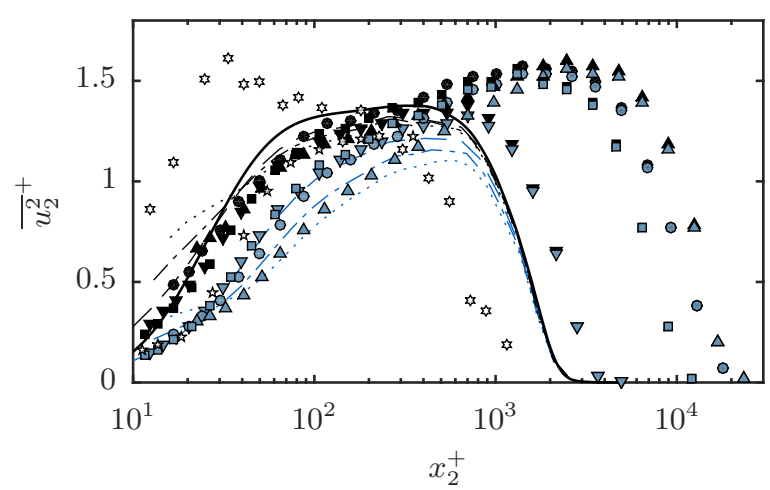

Fig. 6 Inner-normalized existing and present wall-normal velocity variance data acquired by probes which simultaneously measure velocity and vorticity vectors. Dark symbols represent the variance of the signal taken from a single sub-array. Light symbols represent the variance taken from the average $u_{2}$ signal, which is resolved about the probe centroid.

There is a paucity of experimentally measured boundary layer spanwise velocity data in the literature, especially at large Reynolds number. The present spanwise velocity variance profiles are plotted in figure 7 . The dark-filled symbols represent the same data as the light-filled symbols, but processed as individual $\times$-array pairs. These data demonstrate that inclusion of gradient information significantly reduces the $u_{3}$ signal variance close to the wall. This influence is anticipated given that the $u_{3}$-measuring array is sensitive to the $\partial u_{1} / \partial x_{2}$ gradient. The lowest Reynolds number experiment closely matches the corresponding simulation predictions regarding spatial resolution, and reproduces subtle features such as the slight 'plateau' in the profile just interior to its sharp decrease in the outer region. The three largest Reynolds number profiles are distinct from the DNS results. This is to be expected, as the current data along with available well-resolved experimental evidence from Baidya [2015] indicate a strong $\delta^{+}$ dependence in the inner-normalized $u_{3}$ variance, thus limiting the usefulness of comparisons with the lower Reynolds number simulation.

As with the streamwise velocity variance, direct comparisons between the present and existing data sets is difficult owing to the difference in Reynolds number. Thus, a second DNS curve (see table 1) has again been included as a more appropriate reference for the existing data sets of B91 and HA97. The spanwise velocity fluctuation data presented in B91 are uniformly below the approximately matched $\delta^{+}$DNS prediction curve by a considerable margin, while the data presented in HA97 uniformly exceed the same DNS curve.

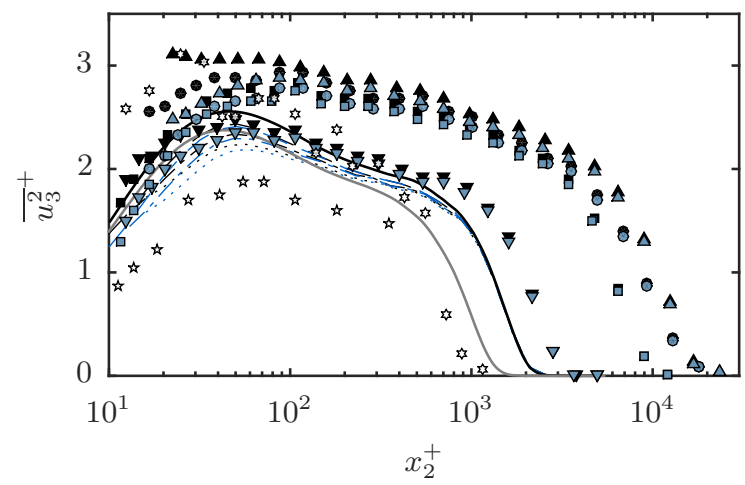

Fig. 7 Inner-normalized existing and present spanwise velocity variance data acquired by probes which simultaneously measure velocity and vorticity vectors. Light-filled symbols are given in table 1. Dark-filled symbols represent the same data as the light-filled symbols with matching shape, but processed as individual $\times$-wire arrays.

The experimental and simulated profile predictions of the $\overline{u_{1} u_{2}}$ Reynolds shear stress, plotted in figure 8 , show very good agreement interior to the logarithmic region. The light and dark-filled symbols represent the covariance of the mean $u_{1}$ and $u_{2}$ signals about the center of the probe, and the mean of the individual covariances of sub-arrays $c$ and $d$, respectively. Again, the covariance of the mean signal is more spatially attenuated, but it is associated with the signal that is best used for correlations or conditional statistics as it is resolved about the centroid of the measurement volume. The experimental results match the simulation predictions for the $\delta^{+} \approx 2600,11000$, and 13200 cases quite well. The cause of the apparent discrepancy in the nearwall region of the $\delta^{+} \approx 7300$ case is unknown at this time.

The Reynolds shear stress data presented in B91 and HA97 are generally quite close the present low Reynolds number individual sub-array data, but are slightly above the present centroid-resolved data in the near-wall region. However, given the sensitivity of this quantity to measurement error, it is not advisable to use the magnitude of the Reynolds shear stress as an analogue for sensor resolution.

The Reynolds shear stress is one of the most difficult quantities to measure, particularly with increasing $\delta^{+}$. This stems from it being constructed from signals whose dominant spectral contributions shift to disparate wavenumbers as $\delta^{+}$becomes large. Accordingly, the magnitude of the $\overline{u_{1} u_{2}}$ profile has a sensitivity to 
probe misalignment in the flow. Operationally, such misalignment can be detected in at least two ways. The first is to observe a proportional increase in $\overline{U_{2}}$ with $\overline{U_{1}}$ in the processed results. The second is to pass freestream calibration data to the lookup tables generated by the jet calibration described in section 2.4.1, and look for the effect of an apparent offset. This offset occurs when the jet calibrator is not perfectly aligned, and was observed to be up to $\pm 2^{\circ}$. This can be corrected by simply subtracting the offset angle globally from the nominal pitch/yaw calibration angles and processing as normal. The pitch and yaw offset values used to process these data sets were determined by comparing the free stream calibration to the jet calibration. Figure 9 shows the sensitivity of the magnitude of the Reynolds stresses (excluding $\overline{u_{2} u_{3}}$ ) to this offset angle at $y^{+} \approx 300$, where the Reynolds shear stress $\overline{u_{1} u_{2}}$ should be close to 1 for all of the present measurements. The trends shown in figure 9 are consistent at all wall-normal locations interior to the wake region. In the wake region all measured Reynolds stresses are less sensitive to the offset angle. The magnitudes of the stresses determined by the pitch sensors, $\overline{u_{1_{c, d}}^{2}}, \overline{u_{2}^{2}}$ and $\overline{u_{1} u_{2}}$, are quite sensitive to alignment error, while those determined by the yaw sensors appear to be less sensitive.

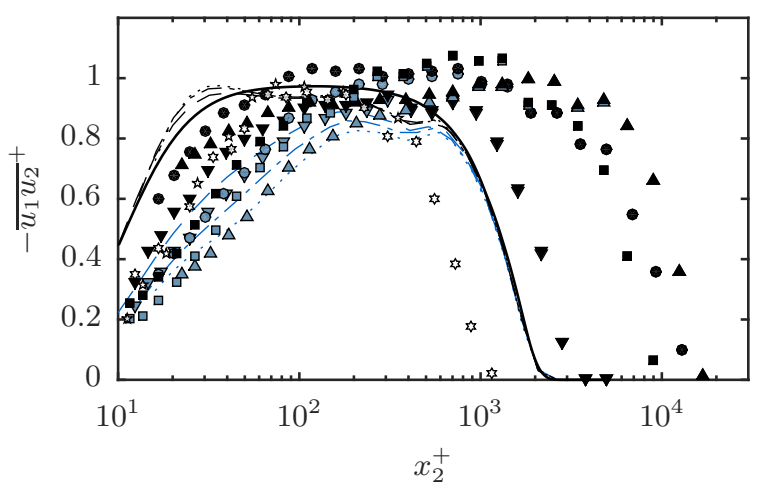

Fig. 8 Inner-normalized existing and present Reynolds shear stress data acquired by probes which simultaneously measure velocity and vorticity vectors. Dark symbols represent the covariance of the signal taken from a single sub-array. Light symbols represent the covariance taken from the average $u_{1} u_{2}$ signal, which is resolved about the probe centroid.

\subsection{Vorticity Statistics}

The RMS profiles of each vorticity component and their constituent gradients are plotted in figures 10 through 16. Data from those studies outlined in section 1 which simultaneously acquired all three components of vortic-
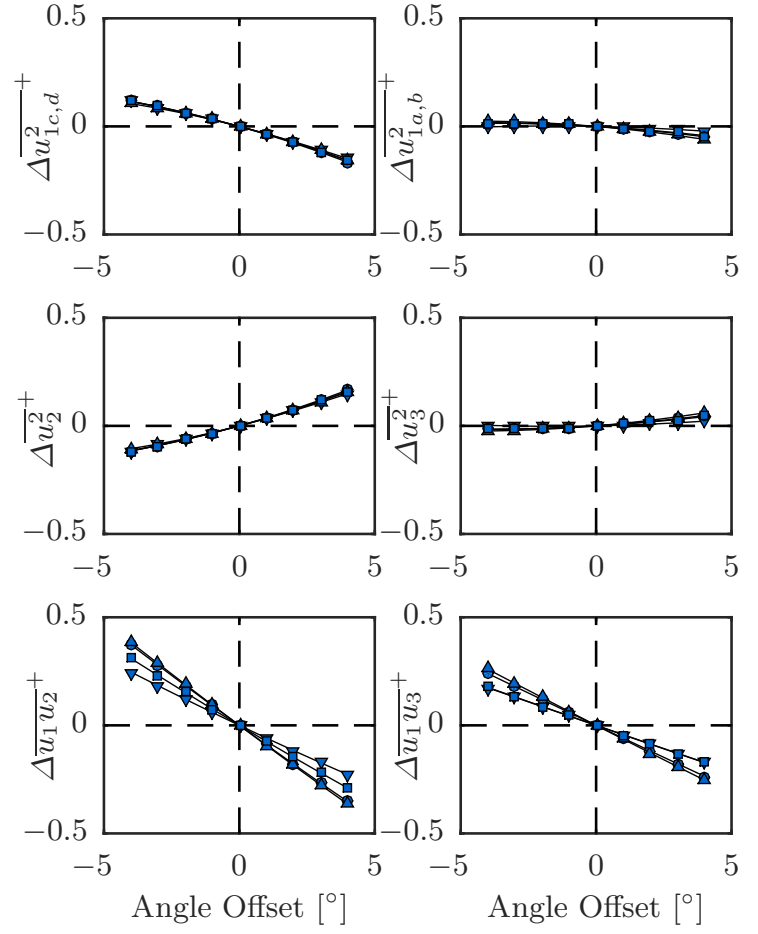

Fig. 9 Sensitivity of inner-normalized Reynolds stresses at $y^{+} \approx 300$ to pitch/yaw calibration misalignment. Positive angles indicate that the nominal 0 angle point in the pitch/yaw calibration was actually producing a positive cross-stream mean flow. Different $\overline{u_{1}^{2}}$ plots correspond to vertical and horizontal sub-array outputs.

ity are summarized in table 1, and are included on the vorticity RMS figures.

\subsubsection{Streamwise Vorticity}

The RMS profiles of $\omega_{1}$ merge under inner-normalization, despite the simulation prediction of decreasing RMS with increasing $l^{+}$. This result apparently contrasts with virtually all other statistics, in which the trends (and often the actual profile magnitudes) are closely predicted by the synthetic probe experiments. The observed disagreement is likely connected to a Reynolds number trend competing with spatial resolution attenuation $\left(\omega_{1}\right.$ is known from DNS to undergo a $\delta^{+}$dependence at low $\delta^{+}$), but based on the agreement between the two matched $l^{+}$cases $(\nabla$ and $\bullet$ ), this does not appear to be the entire story.

The present data are in close agreement with the data of L97, and are generally of similar magnitude to the data of B91. All present data and available comparison data fall below the values of HA97, which exceeds the fully resolved DNS in some regions despite being 
collected at Reynolds number roughly half that of the DNS.

Inspection of the RMS profiles of the constituent gradients of $\omega_{1}$, shown in figures 11 and 12 , reveals very good agreement between experiment and simulation for $\partial u_{2} / \partial x_{3}$. Note that the predicted and actual RMS profiles for the $\partial u_{3} / \partial x_{2}$ gradient are much closer to the fully resolved DNS curve than are those of the $\partial u_{2} / \partial x_{3}$ gradient. This is a result of the prioritization of $x_{2}$ gradients in the probe design. The same disagreement is observed in the $\partial u_{3} / \partial x_{2}$ RMS profile as is observed in the $\omega_{1}$ RMS profile. As the RMS values of $\partial u_{3} / \partial x_{2}$ are generally much larger than those of $\partial u_{2} / \partial x_{3}$, it seems that the observed behavior of $\omega_{1}$ derives from the measured behavior of $\partial u_{3} / \partial x_{2}$. The source of this disagreement in trend between experiment and simulation is thus left unclear. The RMS profiles of the constituent gradients of $\omega_{1}$ for the approximately matched spatial resolution cases appear to merge (to within the scatter of the data) under inner normalization interior to the wake region, suggesting that inner-scaling is appropriate.

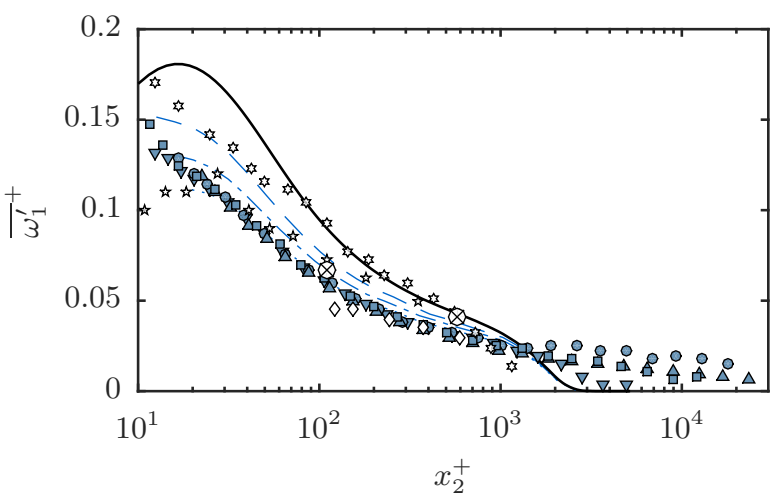

Fig. 10 Inner-normalized existing and present RMS profiles of streamwise vorticity fluctuations and predicted results.

\subsubsection{Wall-normal Vorticity}

The experimental RMS profiles for $\omega_{2}$ show good agreement in trend with the simulation predictions, albeit with slightly lower magnitudes than expected. Interestingly, the RMS profiles for the constituent gradients $\partial u_{1} / \partial x_{3}$ and $\partial u_{3} / \partial x_{1}$ show even better agreement with the simulation predictions. This indicates some degree of deviation from expectation in the covariance between the two constituent gradients. The close agreement between the predicted and actual $\partial u_{3} / \partial x_{1}$ gradient suggests that Taylor's frozen turbulence hypothesis is appropriate (at least in a mean sense) for the spanwise

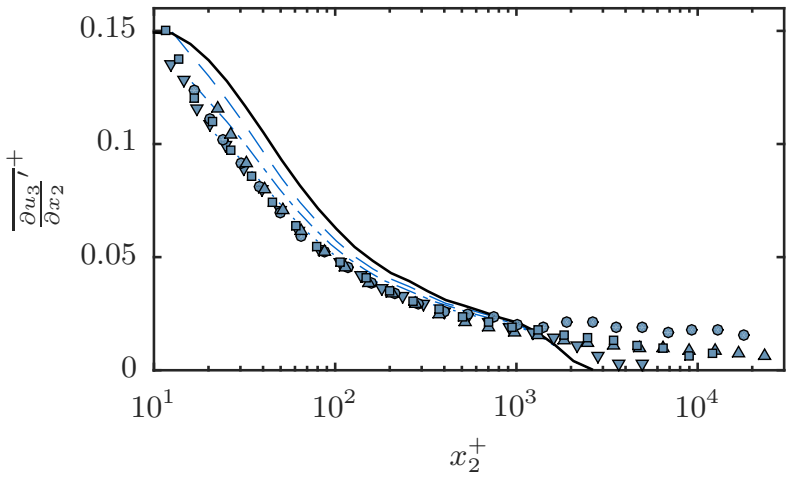

Fig. 11 Inner-normalized RMS of the $\partial u_{3} / \partial x_{2}$ gradient and predicted results.

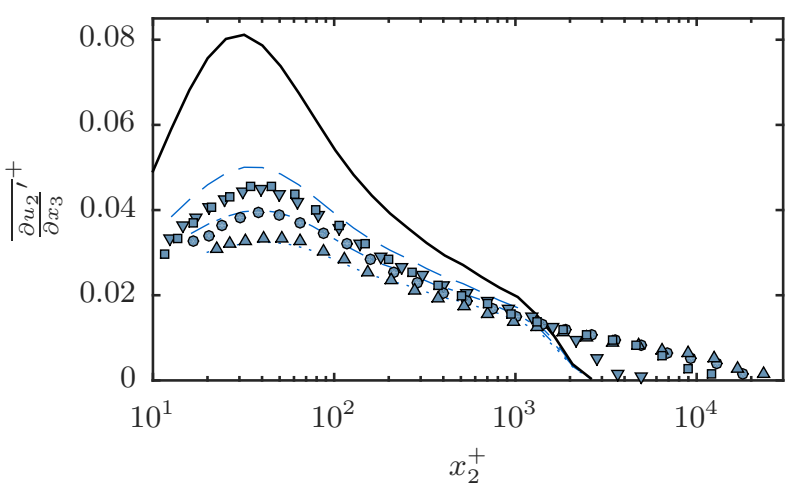

Fig. 12 Inner-normalized RMS of the $\partial u_{2} / \partial x_{3}$ gradient and predicted results.

velocity. The RMS profiles of these gradients for the approximately matched spatial resolution cases also appear to adhere to a single curve under inner normalization interior to the wake region.

The two higher-resolution $\omega_{2}$ RMS profiles of the present effort are in close agreement with the data of L97 and G06. As discussed in section 1, the probe used in L97 made possible a symmetric central difference approach to all gradients, the merits of which are described in Vukoslavčević and Wallace [2013]. This data set was also collected at the highest Reynolds number of all the comparison data, being roughly equal to the lowest Reynolds number of the present data set. When the $\partial u_{1} / \partial x_{3}$ constituent gradient is calculated using the difference between the $u_{1}$ output of sub-arrays $c$ and $d$ (i.e. a central difference relative to the probe centroid), the present RMS profiles lie below the data of B91 and HA97. However, as illustrated in the inset of figure $13(a)$, if the $\partial u_{1} / \partial x_{3}$ constituent gradient is instead calculated from the difference in $u_{1}$ between the probe centroid and either sub-array $c$ or $d$ (i.e. a forward or backward difference relative to the probe cen- 
troid), the present RMS profile increases substantially, to near agreement with the data of B91 and HA97. The RMS data of HA97 are still higher than the present data (regardless of processing) in the outer region of the flow, though it is worth noting that these data also exceed the fully-resolved DNS values, despite their being collected at a Reynolds number substantially lower than that of the DNS. While the increased RMS associated with the forward/backward difference scheme brings the present data closer to the fully resolved DNS curve, it is shown in figure 13(b) to represent a far less accurate measure of the target time-series. The beneficial cancellation of first-order error associated with a central difference scheme clearly outweighs the superior spatial resolution associated with the forward/backward difference scheme in the present case.

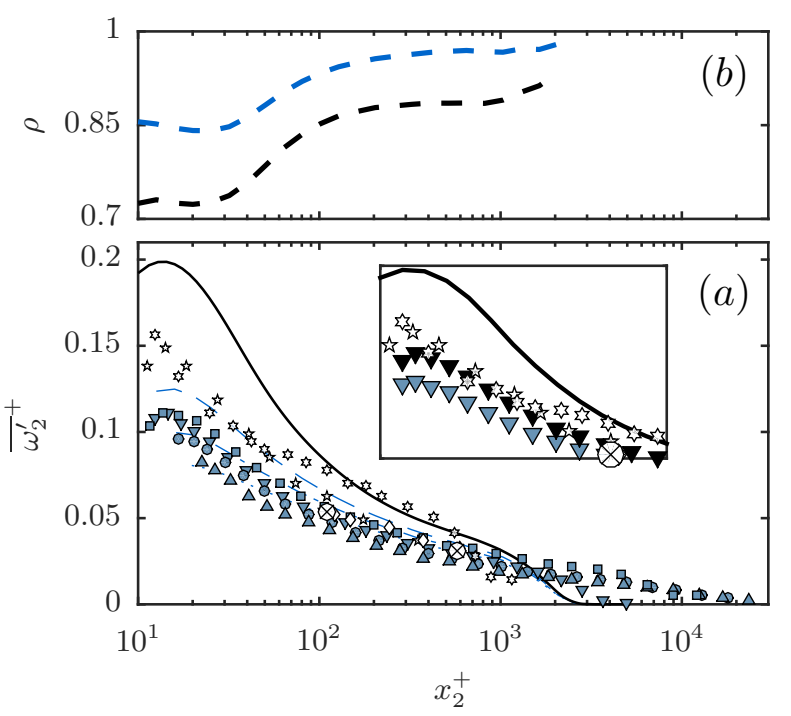

Fig. 13 (a) Inner-normalized existing and present RMS profiles of wall-normal vorticity fluctuations and predicted results. Inset highlights the effects of computing the $\partial u_{1} / \partial x_{3}$ gradient with a forward or backward difference (dark fill) as opposed to a central difference (light fill). (b) Simulated correlation coefficient between the measured $\partial u_{1} / \partial x_{3}$ gradient and its known value at the probe centroid as computed with a forward or backward difference (dark line) as opposed to a central difference (light line). Note that the dark fill symbols in the inset of (a) correspond to the poorer correlation coefficient, despite having RMS values closer to the fully resolved DNS.

\subsubsection{Spanwise Vorticity}

The RMS profiles of $\omega_{3}$ also match the simulation predictions quite well. The predicted trend with $l^{+}$is observed and is in agreement with the trend reported in

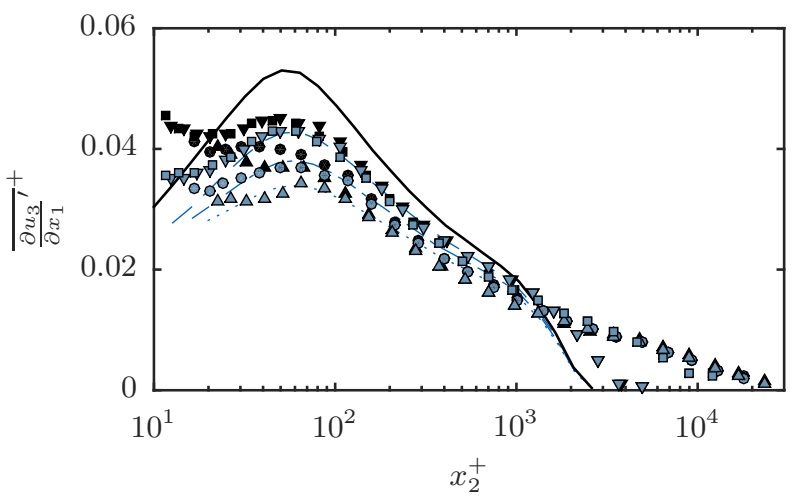

Fig. 14 Inner-normalized RMS of the $\partial u_{3} / \partial x_{1}$ gradient and predicted results. Light-filled symbols represent the profiles summarized in table 1 processed as two 4 -wire gradient arrays. Dark-filled symbols represent the same data as the lightfilled symbols with matching shape, but processed as individual $\times$-wire arrays.

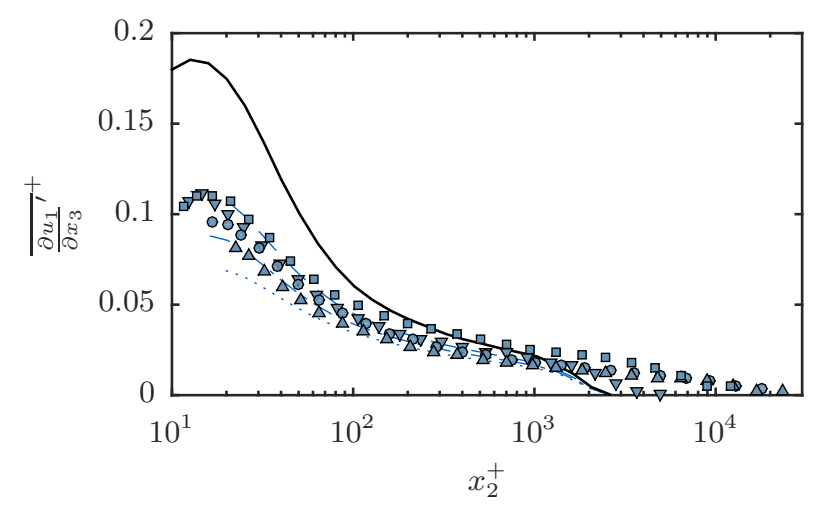

Fig. 15 Inner-normalized RMS of the $\partial u_{1} / \partial x_{3}$ gradient and predicted results.

Morrill-Winter et al. [2015]. The RMS profiles of the constituent gradients of $\omega_{3}$ are shown in figures 17 and 18 , and also show very good agreement with the simulation predictions. In particular, the agreement between the predicted and actual $\partial u_{2} / \partial x_{1}$ RMS profiles for both the individual sub-array and centroid-resolved cases suggest that Taylor's frozen turbulence hypothesis is, at least in a mean sense, appropriate for the wall-normal velocity. The RMS profiles of these gradients for the approximately matched spatial resolution cases also show agreement under inner normalization interior to the wake region.

The present data are in close agreement with the data of B91, L97, and G06 interior to the wake region. The $\omega_{3}$ RMS data of HA97 are higher than the present data as well as the rest of the comparison data over much of the domain.

A further check of the measurement fidelity of $\omega_{3}$ is its skewness, $S_{\omega_{3}}$, plotted in figure 19. It is rational to 


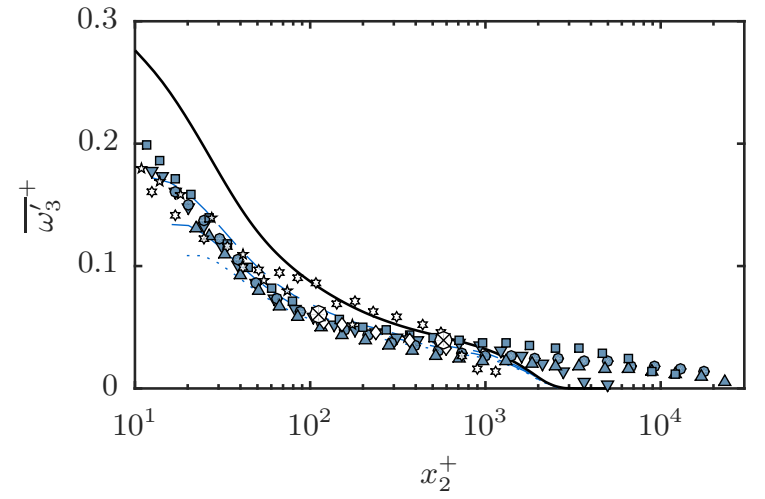

Fig. 16 Inner-normalized existing and present RMS profiles of spanwise vorticity fluctuations and predicted results.

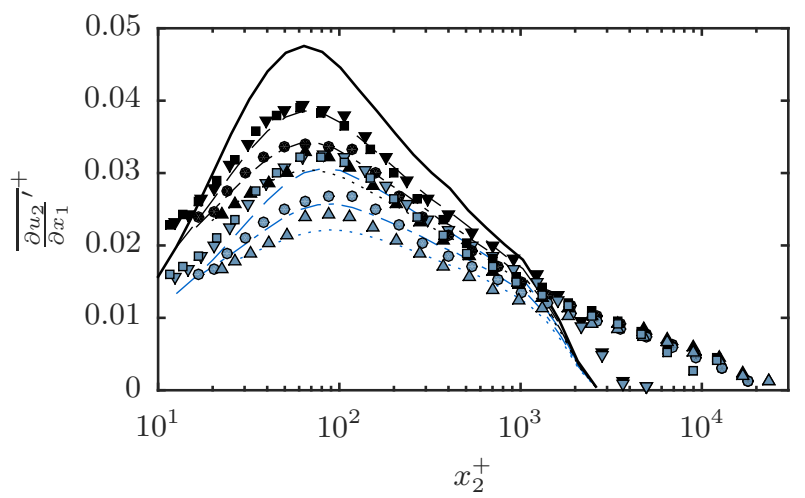

Fig. 17 Inner-normalized RMS of the $\partial u_{2} / \partial x_{1}$ gradient and predicted results. Dark-filled symbols represent the RMS of the signal taken from a single sub-array. Light-filled symbols represent the RMS taken from the streamwise gradient of the average $u_{2}$ signal, which is resolved about the probe centroid.

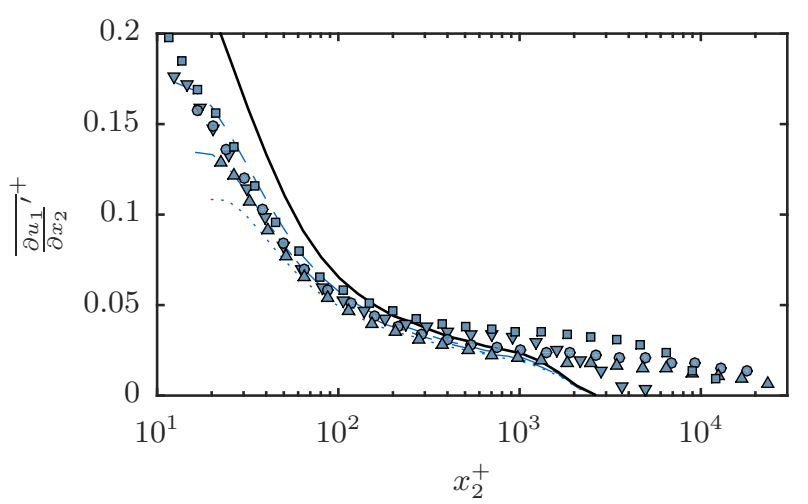

Fig. 18 Inner-normalized RMS of the $\partial u_{1} / \partial x_{2}$ gradient and predicted results.

expect $S_{\omega_{3}}$ to skew toward the sign of $\Omega_{3}$ (negative in this coordinate system) based on the existence of concentrated regions of shear (e.g., as observed by Meinhart and Adrian [1995]). By definition, these regions of shear are characterized by strong intermittent spanwise vorticity of the same sign as the mean. This is indeed what is observed in the present data as well as the DNS of Sillero et al. [2013]. The experimental data match the simulation predictions very well in trend where a spatial resolution effect is predicted, namely interior to the logarithmic region. The simulation results do not show a spatial resolution trend in the logarithmic region. When combined with the predicted behavior of the RMS profile, this indicates that the attenuation that does occur does not impact the vorticity of one sign more than the other at the simulation Reynolds number. This, along with the agreement between the two profiles with approximately matched $l^{+}$, suggests that the agreement observed in the logarithmic region across all measured Reynolds numbers is reflective of the underlying physics of the flow rather than an artifact of imperfect spatial resolution.

The spanwise vorticity skewness data presented in B91 and HA97 did not extend far enough from the wall to capture this trend. Still, where comparison is possible, the present data show close agreement in magnitude and trend with the data of B91. The data of HA97 are of a similar magnitude as the present data, but do not exhibit the same trend.

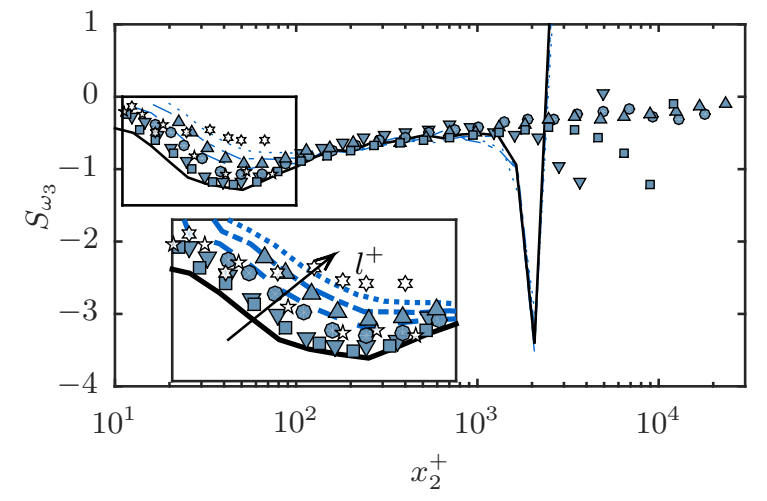

Fig. 19 Existing and present skewness profiles of $\omega_{3}$ and predicted results.

\section{Conclusions}

A multi-sensor hot-wire probe tailored for the simultaneous measurement all three components of velocity and vorticity in boundary layers has been designed, fabricated and tested. The design of this probe was guided by a DNS-based synthetic probe experiment that extends the initial such work of Vukoslavčević et al. [2009] by accounting for finite wire length and non-ideal cooling coefficients. To the author's knowledge, this is the 
first instance that DNS has been used from the start to guide the design and eventual testing of an actual physical probe. The resulting probe yields reliably repeatable data, and, when compared to similarly compact probes, is physically rugged. These attributes are implicitly demonstrated by the quality (smoothness) and data density of the experimental profiles presented herein.

The synthetic experiments were especially useful in quantifying the effects of including gradient information in the data processing, and comparing the accuracy of centered versus non-centered estimates of various gradients and velocity components. It is found that access to gradient information significantly improves the measurement of $u_{3}$ near the wall owing to the undue influence of the $\partial u_{1} / \partial u_{2}$ gradient on the $u_{3}$ signal for a typical horizontal $\times$-wire array. Spanwise gradient information estimated over the width of the probe did not have a noticeable effect on the $u_{2}$ signals produced by the individual vertical $\times$-wire arrays. While this data reduction scheme was only tested on one probe orientation, we tentatively surmise that the effectiveness of including gradient information depends at least on the separation ratio between the two probes used to measure the gradient and the two sensing elements of a single sub-array. In the present case this was 2 for the wires in the $x_{1}-x_{3}$ plane and 5 for the wires in the $x_{1}-x_{2}$ plane.

The probe was deployed in the UNH FPF boundary layer to leverage the large physical scales and low speeds at large $\delta^{+}$. Experiments were conducted over nearly a decade in friction Reynolds numbers. The results of these physical experiments are compared to predictions based on the same synthetic experiment that was used to aid in the probe design and processing scheme evaluation. To the degree to which matching DNS predictions of signal variance indicates measurement quality, the present effort represents the most successful attempt to measure the velocity vector in a turbulent boundary layer by means of a technique also capable of simultaneously measuring the vorticity vector. Even compared to studies which targeted the velocity vector but not the vorticity vector (in a turbulent boundary layer), the present profiles represent some of the largest Reynolds number (well resolved) data in existence. The inner-normalized spatial resolution of the present sensor is shown to result in considerable vorticity component attenuation in the near-wall region. Still, the predicted correlation coefficients and the good agreement generally observed between the experimental results and the simulation predictions (despite the differences in Reynolds number) suggest that much of the time-series information is captured, particularly in the inertial logarithmic and wake regions. In the profile statistics where a magnitude difference is observed between the experiment and the predictions, there is generally agreement in spatial resolution trend. The agreement between the gradient RMS profiles between the matched spatial resolution measurements at $\delta^{+} \approx 2600$ and $\delta^{+} \approx 7300$ suggests that inner-normalization is at least approximately appropriate for shear velocity gradients interior to the wake region.

The synthetic experiment predicts spatial resolution independence in the $\omega_{3}$ skewness in the logarithmic region at a friction Reynolds number of $\delta^{+} \approx 2000$. This prediction, combined with the agreement with all of the experimental data, at both matched and disparate $l^{+}$, suggests that the mixture of positive and negative spanwise vorticity fluctuations is essentially independent of Reynolds number. The skewness profile also appears to follow an approximately logarithmic trend in the region where the mean velocity profile is logarithmic. This behavior is hypothesized to be related to the changes in strength and/or spatial organization, with changing distance from the wall, of the internal shear layers that are known to exist. Elucidation of this relationship, however, requires further investigation.

Acknowledgements The authors wish to gratefully thank the financial support of the Australian Research Council and the USA National Science Foundation.

\section{References}

R. A. Antonia, T. Zhou, and Y. Zhu. Three-component vorticity measurements in a turbulent grid flow. $J$. Fluid Mech., 374:29-57, 1998.

R. Baidya. Multi-component velocity measurements in turbulent boundary layers. $\mathrm{PhD}$ thesis, University of Melbourne, Department of Mechanical and Manufacturing Engineering, 2015.

O. M. Bakken and P.A. Krogstad. A velocity dependent effective angle method for calibration of x-probes at low velocities. Exp. Fluids, 37(1):146-152, 2004.

J.-L. Balint, P. V. Vukoslavičević, and J. M. Wallace. A study of the vortical structure of the turbulent boundary layer. In Advances in Turbulence, pages 456-464. Springer, 1987.

J.-L. Balint, J. M. Wallace, and P. V. Vukoslavičević. The velocity and vorticity vector fields of a turbulent boundary layer. part 2. statistical properties. J. Fluid Mech., 228:53-86, 1991.

P. Bradshaw. An introduction to turbulence and its measurement. Pergamon Press, Oxford, 1971.

H. H. Bruun. Hot-wire anemometry-principles and signal analysis. Oxford Science Publications, 1995. 
K. A. Chauhan, P. A. Monkewitz, and H. M. Nagib. Criteria for assessing experiments in zero pressure gradient boundary layers. Fluid Dynam. Res., 41(2): 021404, 2009.

K. Dobbeling, B. Lenze, and W. Leuckel. Basic considerations concerning the construction and usage of multiple hot-wire probes for highly turbulent threedimensional flows. Measurement Science and Technology, 1(9):924, 1990.

R. Ebner. Influences of roughness on the inertial mechanism of turbulent boundary-layer scale separation. PhD thesis, 2014.

B. Ganapathisubramani, E. K. Longmire, and I. Marusic. Experimental investigation of vortex properties in a turbulent boundary layer. Physics of Fluids, 18 (5):055105, 2006.

A. Honkan and Y. Andreopoulos. Vorticity, strain-rate and dissipation characteristics in the near-wall region of turbulent boundary layers. J. Fluid Mech., 350: 29-96, 1997.

N. Hutchins and I. Marusic. Evidence of very long meandering features in the logarithmic region of turbulent boundary layers. J. Fluid Mech., 579:1-28, 2007.

F. E. Jorgensen. Directional sensitivity of wire and fiber-film probes. DISA Information, 11(3):1-7, 1971.

J. C. Klewicki and R. E. Falco. On accurately measuring statistics associated with small-scale structure in turbulent boundary layers using hot-wire probes. $J$. Fluid Mech., 219:119-142, 1990.

R. E. Lawson and R. E. Britter. A note on the measurement of transverse velocity fluctuations with heated cylindrical sensors at small mean velocities. J. Phys. E, 16(6):563, 1983.

G. C. Lemonis. An experimental study of the vector fields of velocity and vorticity in turbulent flows. $\mathrm{PhD}$ thesis, 1995.

G. C. Lemonis. Three-dimensional measurement of velocity, velocity gradients and related properties in turbulent flows. Aerospace science and technology, 1(7):453-461, 1997.

P. M. Ligrani and P. Bradshaw. Spatial resolution and measurement of turbulence in the viscous sublayer using subminiature hot-wire probes. Exp. Fluids, 5 (6):407-417, 1987.

I. Marusic, J. P. Monty, M. Hultmark, and A. J. Smits. On the logarithmic region in wall turbulence. J. Fluid Mech., 716:R3, 2013.

C. D. Meinhart and R. J. Adrian. On the existence of uniform momentum zones in a turbulent boundary layer. Phys. Fluids, 7(4):694-696, 1995.

M. M. Metzger and J. C. Klewicki. A comparative study of near-wall turbulence in high and low Reynolds number boundary layers. Physics of Fluids, 13(3):
692-701, 2001.

C. Morrill-Winter. Structure of mean dynamics and spanwise vorticity in turbulent boundary layers. $\mathrm{PhD}$ thesis, 2016.

C. Morrill-Winter, J. Klewicki, R. Baidya, and I. Marusic. Temporally optimized spanwise vorticity sensor measurements in turbulent boundary layers. Exp. Fluids, 56(12):1-14, 2015.

L. Ong and J. M. Wallace. Joint probability density analysis of the structure and dynamics of the vorticity field of a turbulent boundary layer. Journal of Fluid Mechanics, 367:291-328, 1998.

S.-R. Park and J. M Wallace. The influence of instantaneous velocity gradients on turbulence properties measured with multi-sensor hot-wire probes. Exp. Fluids, 16(1):17-26, 1993.

A. Schröder, R. Geisler, G. E. Elsinga, F. Scarano, and U. Dierksheide. Investigation of a turbulent spot and a tripped turbulent boundary layer flow using timeresolved tomographic piv. Experiments in Fluids, 44 (2):305-316, 2008.

J. A. Sillero, J. Jiménez, and R. D. Moser. Onepoint statistics for turbulent wall-bounded flows at reynolds numbers up to $\delta^{+} \approx 2000$. Phys. Fluids, 25 (10):105102, 2013.

W. H. Snyder and I. P. Castro. The yaw response of hot-wire probes at ultra-low wind speeds. Meas. Sci. Tech., 9(9):1531, 1998.

H. Tennekes and J. L. Lumley. A first course in turbulence. The MIT Press, 1972.

A. A. Townsend. Structure of turbulent shear flow. Cambridge University Press, 1976.

A. Tsinober, E. Kit, and T. Dracos. Experimental investigation of the field of velocity gradients in turbulent flows. J. Fluid Mech., 242:169-192, 1992.

C. W. H. Van Doorne and J. Westerweel. Measurement of laminar, transitional and turbulent pipe flow using stereoscopic-piv. Experiments in Fluids, 42(2):259279, 2007.

P. Vincenti, J. Klewicki, C. Morrill-Winter, C. M. White, and M. Wosnik. Streamwise velocity statistics in turbulent boundary layers that spatially develop to high reynolds number. Exp. Fluids, 54(12):1629, 2013.

P. V. Vukoslavčević and J. M. Wallace. Influence of velocity gradients on measurements of velocity and streamwise vorticity with hot-wire $\mathrm{x}$-array probes. Rev. Sci. Inst., 52(6):869-879, 1981.

P. V. Vukoslavčević and J. M. Wallace. The influence of the arrangements of multi-sensor probe arrays on the accuracy of simultaneously measured velocity and velocity gradient-based statistics in turbulent shear flows. Experiments in fluids, 54(6):1537, 2013. 
P. V. Vukoslavčević, N. Beratlis, E. Balaras, J. M. Wallace, and O. Sun. On the spatial resolution of velocity and velocity gradient-based turbulence statistics measured with multi-sensor hot-wire probes. Exp. Fluids, 46(1):109-119, 2009.

J. M. Wallace and P. V. Vukoslavčević. Measurement of the velocity gradient tensor in turbulent flows. Ann. Rev. Fluid Mech., 42:157-181, 2010.

B. W. Zeff, D. D. Lanterman, R. McAllister, R. Roy, E. J. Kostelich, and D. P. Lathrop. Measuring intense rotation and dissipation in turbulent flows. Nature, 421(6919):146, 2003.

T. Zhou, Y. Zhou, M. W. Yiu, and L. P. Chua. Threedimensional vorticity in a turbulent cylinder wake. Exp. Fluids, 35(5):459-471, 2003. 


\section{University Library}

\section{- M M N E R VA A gateway to Melbourne's research publications}

Minerva Access is the Institutional Repository of The University of Melbourne

Author/s:

Zimmerman, S;Morrill-Winter, C;Klewicki, J

Title:

Design and implementation of a hot-wire probe for simultaneous velocity and vorticity vector measurements in boundary layers

Date:

2017-10-01

Citation:

Zimmerman, S., Morrill-Winter, C. \& Klewicki, J. (2017). Design and implementation of a hotwire probe for simultaneous velocity and vorticity vector measurements in boundary layers. EXPERIMENTS IN FLUIDS, 58 (10), https://doi.org/10.1007/s00348-017-2433-8.

Persistent Link:

http://hdl.handle.net/11343/283163 